\title{
Is Depression a Social and Reactive Phenomenon to Parkinson's (PD) or an Internal Symptom of the Disease Itself?
}

Received: January 28, 2019; Accepted: February 19, 2019; Published: February 25, 2019

\section{Introduction}

Depression is a common condition in patients with (PD). To date, numerous contradictory studies have been published about whether depression is really a reactive manifestation of PD (because of the inability generated by movement problems) or an associated and endogenous component due to a common aetiology.

Answering this question is vital since, when in conjunction with PD, treatments for depression will vary depending upon its origin. Why is it so difficult to answer this question? There are some ideas to consider:

\section{Brief historical context of the issue}

A-The diagnosis of depression in PD is difficult since most symptoms of depression (motor and non-motor) such as lethargy, decreased concentration, sleep disturbance, fatigue and loss of strength (among others), can also be characteristic of PD.

Even, sometimes, the most skeptical opinions support the absence of depression in PD, arguing that the mentioned symptoms belong, only, to this last disease. Consequently, feelings of sadness or apathy in patients with PD would not be as deep as to talk about depression.

However, in the 1990s there was a discovery that ensured the simultaneous existence of depression and EP: similar decreases in serotonin and other amines -such as dopamine and noradrenaline- had been found between patients with primary depression and some subjects who was believed suffering only from PD. Therefore, these concordances showed that sadness or anhedonia could be felt so deep to affirm that depression existed in comorbidity with PD.

Based on these results, we could only ensure comorbidity but we could not answer which of these two diseases originates the other one.

B- However, even though all patients with PD suffer a decrease in these neurotransmitters, not all patients with PD end up suffering from depression. This led some neurologists and neuropsychologists to question the endogenous origin of the later and conclude that depression was only a reactive manifestation in a few subjects due to social adaptation problems.

\section{Santiago Benito* \\ Department of Philosophy and Psychology, Ministry of Education of Madrid, Spain}

* Corresponding authors: Santiago Benito

” santibenito8@gmail.com

Tel: 0034689148385

Professor, Department of Philosophy and Psychology, Ministry of Education of Madrid, Spain.

Citation: Benito S (2019) Is Depression a Social and Reactive Phenomenon to Parkinson's (PD) or an Internal Symptom of the Disease Itself? J Transl Neurosci Vol.4 No.1:2

Despite this, in the last decade, not all scientists agreed because there was evidence of patients with higher damage of PD but who did not suffer from depression. According to their view, if depression were a reaction to $P D$, patients with more damage in their movement would have deeper depressions. This situation is not corroborated empirically: the degrees on depression oscillate regardless of the social damage caused by PD.

The defenders of the reactive hypothesis affirmed that there were other variables (in addition to the motor damage) which would explain the non-correspondence between a higher degree of depression and a higher damage due to PD: family support, personality of the subject ... So, It should be the defenders of the endogenous theory who explain why not all patients with decreases in amine levels suffer depression.

C) Despite the many studies that have aimed to find the answer there are not any conclusive results to date: the general opinion is that all patients with PD have depression, but it's not strong enough to be detected in all cases.

However, according to more current studies by Marsden $\mathrm{C}$ and others, the comorbidity between both diseases is $46 \%$, resulting in $54 \%$ of patients with PD presenting no depression. This figure is too high to prove that depression exists without detection.

The defenders of the common aetiology arguing, in turn, that there are cases in which emotional symptoms go unnoticed when a PD patient are diagnosed, (between 10 and 25 percent) [1]. But, even if this figure was true, there would still be a large number of patients with PD but not depression. 
Parallel to this, new data compiled in recent years has led scientists to think of depression being a link to Parkinson as an "early precursor" as patients with a previous diagnosis of depression are twice as likely to develop PD (up to 3 and 6 years later) when compared to those who have not experienced depression. To respond to the attacks received by the defenders of the reactive theory, it has been assumed that there may be cases in which this precursor depression disappears after the appearance of PD because it is replaced.

So, these cases where depression is "an early ancestor of PD" would show how both pathologies have a common aetiology that makes them indiscernible as different diseases. However, in the absence of real and valid results, the debate is still open.

\section{Latest and novel contributions}

In orden to respond to those unknowns we will use a study by Serrano- Dueñas, M [2] in which he tried to find the relationship between depression, its intensity and motor disability. The main hypothesis was to check whether subjects, with PD whose dominant side of the brain was primarily affected, had higher depressive rates than those affected with the non- dominant side. If it is affirmative, it could be considered that depression is an associated reaction to the practical disabilities produced by EP.

The first tests carried out using ADLS and UPDRSm were performed on 63 patients with right unilateral PD (divided into two groups: dominant right side and non-dominant right side). The results did not find significant differences, showing a higher functional damage and a motor disability in patients whose right side was dominant - for writing activities, adjusting screws and stringing a thread on a needle. This fact eliminates depression as a reactive phenomenon and corroborates that it is an endogenous manifestation and not a secondary depression linked to PD.

However, a second part of the study indicated that those patients affected on its dominant side (right) had a higher index of depression than those patients whose affected right side was not dominant. Members of both groups were treated with L-dopa and, also, with other drugs that could reestablish the level of amines. Different patients, with the same level of depression,

\section{References}

1 MMartínez MC, López M (2002) Diagnóstico diferencial entre depresión, enfermedad de Parkinson y síndromes parkinsonianos (parkinsonismos). Revista Medicina General 45: 514-518. did not receive that treatment. The results showed no significant changes between those medicated patients and who were not. The amines did not seem to be related-actually- to depression.

\section{Can we talk about a common aethology not associated with amines?}

Yes, because the common cause of both diseases is not related to neurotransmitters but with neuroanatomical bases:

The studies carried out by Dormondt, D [3] had the objective of stimulating with electrodes the left black substance of a PD patient. The result was an acute depressive symptomatology while this stimulation was applied.

This fact confirmed- using a positron emission tomography- the identification of two damaged circuits. Two neuronal connections participated- simultaneously - in the neurobehavioral disorders observed in PD and mood symptoms of depression. These circuits were the orbitofrontal circuit and the dorsolateral circuit. Both pass together, as we know, through the frontal lobes and subcortical structures.

This finding concluded that the pathways which pass through the left black substance or those Ares which are influenced by it, May are related to the origin of depression, PD and their comorbidity when they are stimulated or when they are inhibited.

To sum up, it is possible that the ignorance about these two common pathways has led to a large percentage of misdiagnoses in which depression went unnoticed and all symptoms were attributed to PD. In a similar way, it would be a mistake to consider some of common symptoms only for depression, when they should be adjudicated to PD too.

After all, concomitant depression with PD has an endogenous aetology because it is characterized by a damaged and common neuroanatomical structure that differs from the simple mismatch of neurotransmitters. In this case, there could be a decrease in amines but not a depression if we take into account that orbitofrontal and dorsolateral circuit are not damaged by genetic issues or other reasons still to be studied.

2 Serrano-Dueñas M (2000) Enfermedad de Parkinson, hemicuerpo afectado y depresión. Revista Neurol 31: 1109-1112.

3 Bejjani BP, Damier P, Arnulf I, Thivard L, Bonnet AM, et al. (1999) Transient acute depression induced by high-frequency deep-brain stimulation. N Engl J Med 340: 1476-1480. 